\title{
Thermal Support and Rapid Uniform Rotation: Implications on Protoneutron Stars, Hot Neutron Stars, and Neutron Star Merger Remnants
}

\author{
P.S. Koliogiannis, Ch.C. Moustakidis \\ Department of Theoretical Physics, Aristotle University of Thessaloniki, \\ 54124, Thessaloniki, Greece
}

Received 15 October 2021

doi: https://doi.org/10.55318/bgjp.2021.48.5-6.547

\begin{abstract}
The equation of state along with the hot neutron star matter provides an important framework for studying essential astrophysical phenomena, such as the formation of protoneutron stars and the neutron star merger remnants. The equations of state of the fluid in the interior of the star for the above dynamical phenomena are based on the momentum-dependent interaction model and state-of-the-art microscopic data. In particular, we construct them at finite temperature with beta-stable matter, and finite entropy per baryon with varying proton fractions. Furthermore, we investigate in detail the thermal and rotation with the Kepler frequency effects on neutron star quantities, including the mass and radius, the frequency, the Kerr parameter, the moment of inertia, the central baryon density, etc. Thermal support and its effect on isolated neutron stars, as well as on the postmerger remnants, could provide useful insight and robust constraints on the equation of state of nuclear matter.
\end{abstract}

KEY WORDS: nuclear matter, hot and dense matter, neutron stars, binary neutron star merger remnant.

\section{Introduction}

Protoneutron stars are formed in the aftermath of the gravitational collapse of the core of a massive star. At this stage, the star begin its life with a large amount of thermal energy and neutrinos trapped in the interior of the star [1-4]. On the other hand, hot neutron stars are formed as a result of mass accretion due to a companion (absence of neutrinos). Both of them are crucial for astrophysical phenomena, such as the neutron star mergers, the formation of protoneutron 
stars, and the formation of black holes. An important ingredient for the study of hot and dense matter is the equation of state (EoS) and its associated neutrino opacities, which at high densities (several times the normal nuclear matter density) remains unknown.

At the moment, observations are the most powerful tool to impose constraints on the high density properties of the EoS. In particular, both static and rotating neutron stars are the main object of observations. The most robust ones are the maximum mass, including the resent PSR J0740+6620 with $M=2.14_{-0.09}^{+0.10} M_{\odot}$ [5], and the spin frequency of a neutron star, including the PSR J1748-244ad with $f=716 \mathrm{~Hz}$ [6]. An additional source of constraints are the observations of binary neutron star mergers. Specifically, the observations of the GW170817 [7] and GW190425 [8] events, have contributed to the unknown intermediate region of densities. Concerning the high density region, reliable candidates are the remnant and its maximum stable mass, spin period, and lifetime, which are strongly depend on the dense matter properties at high temperature and entropy.

In the present work we construct a set of thermodynamically consistent isothermal and isentropic EoSs, based on the parameterized cold one, and apply them in order to study the bulk properties of hot neutron stars. In particular, we study the importance of the free energy and the effect of the proton fraction related to the cooling through dynamical processes. We examine the evolution of the neutron star through the sequences of constant baryon mass, connecting macroscopic with microscopic properties. Furthermore, we focus on the extensive study on some of the bulk properties of both nonrotating and rotating at the mass-shedding limit hot neutron stars, including the moment of inertia, Kerr parameter, and ratio of rotational kinetic to gravitational binding energy $T / W$. Finally, we study the hot, rapidly rotating remnant of a binary neutron star merger related to the EoSs. For an extensive study and information about the construction of the EoSs and their applications see Ref. [9].

The paper is structured as follows. In Section 2 we present the EoS at finite temperature, and in particular the constructive model and the thermodynamical quantities, while in Section 3 we display the astrophysical implications. The scientific remarks are contained in Section 4.

\section{Equation of State at Finite Temperature}

\subsection{Momentum-dependent model and construction of the equation of state}

The EoSs for the description of hot neutron star matter are based on the momentum-dependent interaction (MDI) model [10-12] and data provided by Akmal et al. [13] for the APR-1 EoS (hereafter MDI+APR1). In particular, the schematic presentation of the cold MDI+APR1 EoS for the symmetric nuclear 


\section{Thermal Support and Rapid Uniform Rotation}

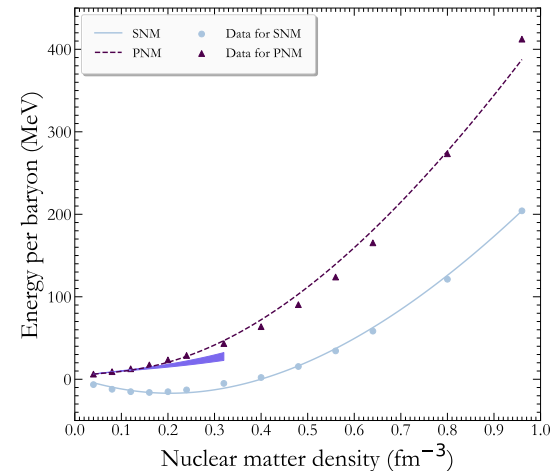

Figure 1. (Color online) The fits of SNM and PNM of MDI+APR1 cold EoS. The SNM is presented by the circles and solid line, while the PNM is presented by the triangles and dashed line. The shaded region corresponds to benchmark calculations of the energy per particle of PNM extracted from Piarulli et al. [17].

matter (SNM) and the pure neutron matter (PNM), as well as the data of Akmal et al. [13] for comparison, are displayed in Figure 1.

The above parameterization for the cold, catalyzed matter is applied for the additionally construction of 10 hot EoSs based on temperatures in the range $[1,60] \mathrm{MeV}$, and nine hot EoSs based on lepton fractions and entropies per baryon in the ranges $[0.2,0.4]$ and $[1,3] k_{B}$, respectively. Furthermore, for the solid crust region, we adopted the EoSs of Feynman et al. [14] and Baym et al. [15], while for the finite temperature cases and the low-density region $\left(n_{b} \leq 0.08 \mathrm{fm}^{-3}\right)$, as well as the finite entropies per baryon and lepton fractions, the EoSs of Lattimer and Swesty [16] and the specific model corresponding to the incomprehensibility modulus at the saturation density of SNM $K_{s}=220 \mathrm{MeV}$ are used.

\subsection{Effects of thermodynamical quantities: free energy and proton fraction}

The free energy per particle is a quantity related to the calculation of the proton fraction. In Figure 2 we display the free energy per particle as a function of the baryon density for (a) the PNM and (b) the SNM and temperatures in the range $[0,60] \mathrm{MeV}$. Although the temperature effect is significant at low densities, at high densities, since the Fermi energy is appreciably higher compared to the thermal energy $k_{B} T$, it is less pronounced, leading to the converge of the free energy-density trends.

Furthermore, in Figure 3 we display the proton fraction as a function of the baryon density for temperatures in the range $[0,60] \mathrm{MeV}$. The introduction of temperature has as a result the appearance of important thermal effects at low 

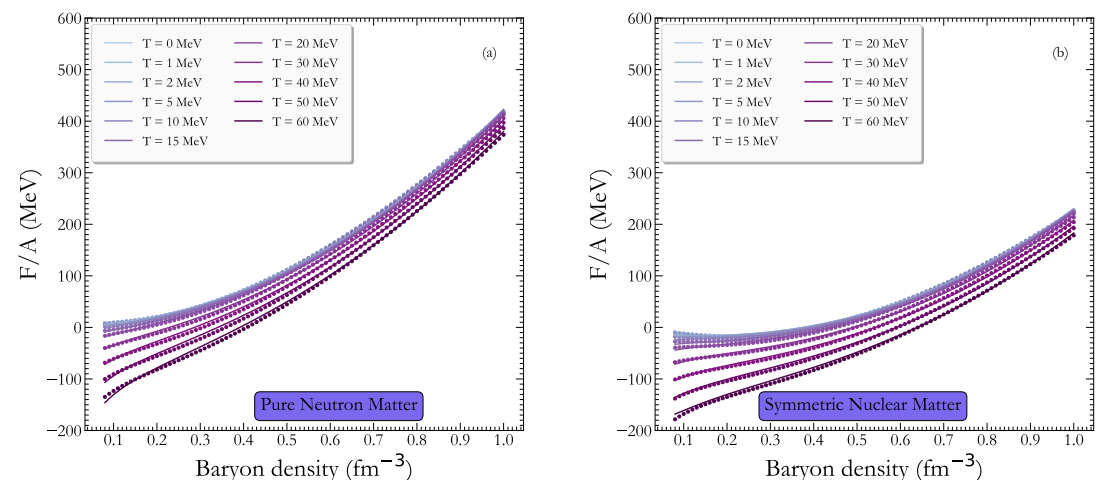

Figure 2. (Color online) The free energy per particle as a function of the baryon density for (a) PNM and (b) SNM and temperatures in the range $[0,60] \mathrm{MeV}$. Fits are presented with the solid lines and data with circles (see also Ref. [9]).

densities, where at high densities the effects are moderated. It has to be noted here that the above dependence is a consequence of the similar sensitivity of free energy per particle on the temperature and it is reflected on the structure of neutron stars [9].

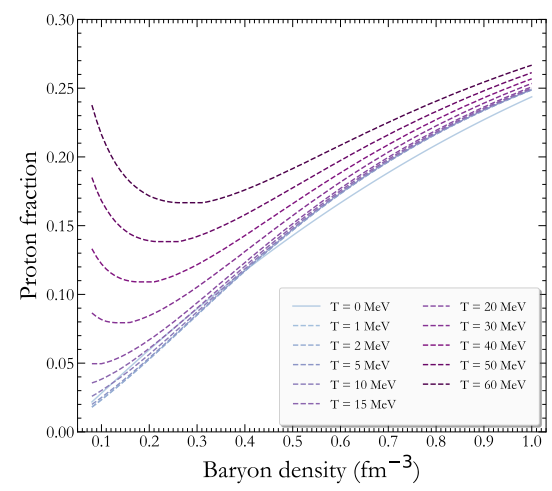

Figure 3. (Color online) The proton fraction as a function of the baryon density for temperatures in the range $[0,60] \mathrm{MeV}$. The cold configuration is presented with the solid line, while the hot ones with dashed lines.

\section{Astrophysical Implications}

\subsection{Sequences of constant baryon mass}

As sequences of constant baryon mass represent the evolution of isolated neutron stars, we applied them in hot neutron stars in order to study thermal effects 

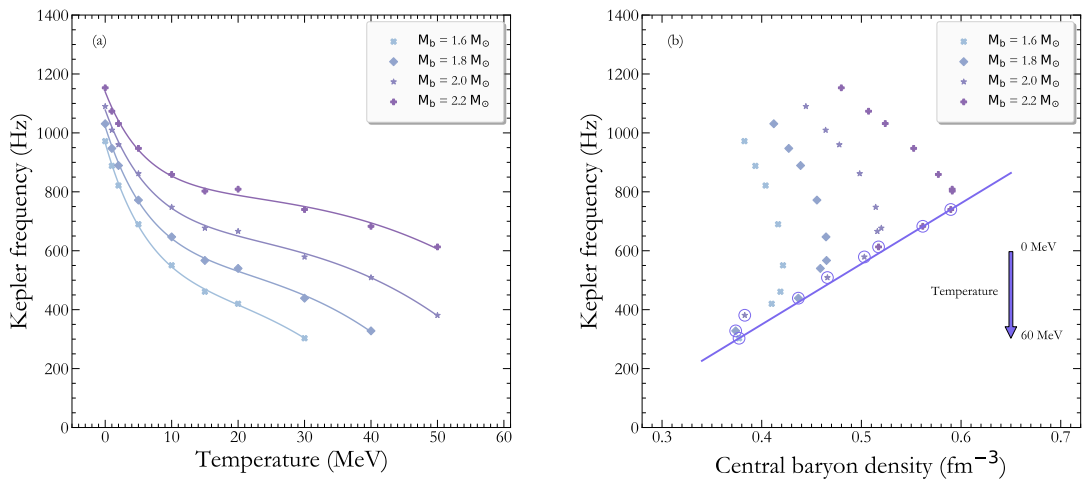

Figure 4. (Color online) The Kepler frequency as a function of (a) the temperature and (b) the central baryon density for constant baryon mass sequences. (a) Solid lines correspond to fits (for more details see Ref. [9]). (b) The solid line corresponds to Eq. (1) and open circles mark the high-temperature region $(T \geq 30 \mathrm{MeV})$.

on the evolution. Specifically, we have used the EoSs in the temperature range $[0,60] \mathrm{MeV}$, and a sequence related to the cooling of the neutron star is constructed. Figure 4 displays the Kepler frequency as a function of (a) the temperature and (b) the central baryon density for four baryon mass configurations in the range $[1.6,2.2] \mathrm{M}_{\odot}$.

In particular, in Figure 4(a) is presented the reduction of the Kepler frequency along the temperature, where for low temperatures, $[0,15] \mathrm{MeV}$, the effect is more pronounced. Furthermore, in Figure 4(b) two distinct features stand out. Firstly, the linear relation that exist for temperatures lower than $30 \mathrm{MeV}$, among the baryon masses and assuming constant temperature. Secondly, and more important, the linear relation that holds on between the Kepler frequency and the central baryon density for temperatures $T \geq 30 \mathrm{MeV}$ and independently of the baryon mass. The relation can be expressed as

$$
f\left(n_{b}^{c}\right)=-473.144+2057.271 n_{b}^{c}, \quad(\mathrm{~Hz})
$$

where the frequency and the central baryon density are given in units of $\mathrm{Hz}$ and $\mathrm{fm}^{-3}$, respectively. The above relation can actually define the allowed region for a hot neutron star rotating at its mass-shedding limit [9].

\subsection{Bulk neutron star properties: moment of inertia, Kerr parameter, and $\mathrm{T} / \mathrm{W}$ ratio}

In Figures 5(a,b) we display the dimensionless moment of inertia as a function of the compactness parameter ${ }^{1}$ for (a) temperatures in the range $[0,60] \mathrm{MeV}$

\footnotetext{
${ }^{1}$ Compactness parameter: $\beta=G M / R c^{2}$.
} 


\section{P.S. Koliogiannis, Ch.C. Moustakidis}
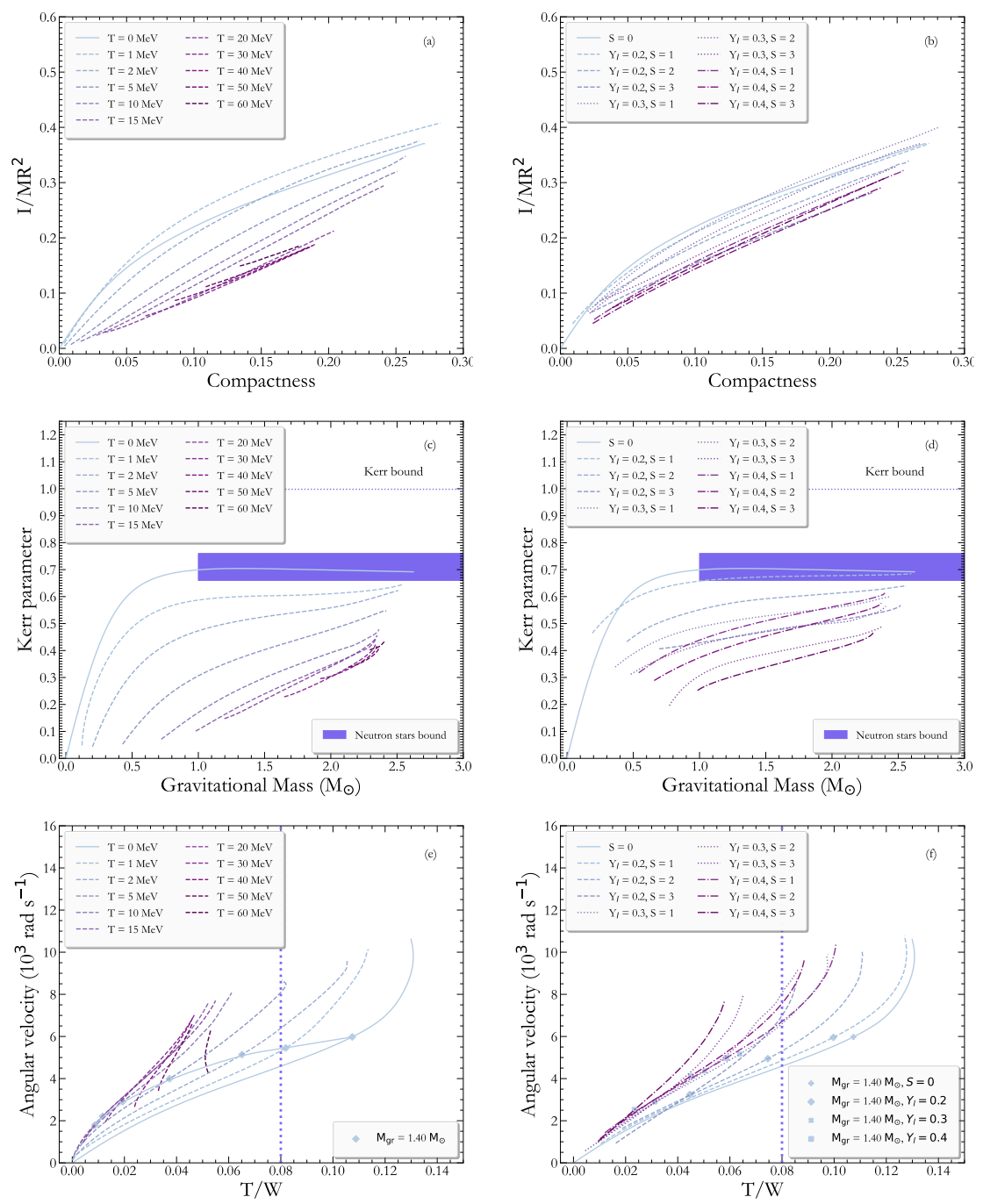

Figure 5. (Color online) (a,b) The dimensionless moment of inertia as a function of the compactness parameter. (c,d) The Kerr parameter as a function of the gravitational mass. The horizontal dotted line marks the Kerr bound for astrophysical Kerr black holes, $\mathcal{K}_{\text {B.H. }}=0.998$ [18]. The shaded region represents the limits for neutron stars from Eq. (3). (e,f) The angular velocity as a function of the ratio of rotational kinetic to gravitational binding energy. The vertical dotted line marks the critical value, $T / W=0.08$ [21], for gravitational radiation instabilities. Plus sign, diamonds, squares, and crosses represent the $M_{\mathrm{gr}}=1.4 M_{\odot}$ configuration. Figures (a,c,e) correspond to isothermal profile, while Figures (b,d,f) correspond to isentropic profile. For all Figures, the cold configuration is presented with the solid line, while hot configurations with the dashed ones. All Figures correspond to the mass-shedding limit. 


\section{Thermal Support and Rapid Uniform Rotation}

and (b) lepton fractions and entropies per baryon in the ranges $[0.2,0.4]$ and $[1,3] k_{B}$, respectively. Dimensionless moment of inertia, in connection with the compactness parameter, could provide crucial information about the structure of the neutron star. Specifically, the increase of temperature or the entropy per baryon in the interior of the neutron star leads to lesser compact objects than the cold neutron star.

Kerr parameter is related both to neutron stars and Kerr black holes, defined via the relation

$$
\mathcal{K} \equiv \frac{c}{G} \frac{J}{M^{2}},
$$

and it is also an indicator of the final fate of the collapse of a rotating compact star. Except from the constraint for Kerr black holes [18], constraints for neutron stars are possible via the relations for angular velocity and moment of inertia in Refs. [19,20]. In particular, we found that the Kerr parameter, at the mass-shedding limit for a cold, catalyzed neutron star, is given by the universal expression

$$
\mathcal{K}_{\mathrm{k}} \simeq 1.34 \sqrt{\beta_{\max }}
$$

Assuming that, for the majority of realistic cold EoSs, the relation $0.24 \leq$ $\beta_{\max } \leq 0.32$ holds, we concluded that $0.66 \leq \mathcal{K}_{\mathrm{k}} \leq 0.76$ [9].

In Figures 5(c,d) we display the Kerr parameter as a function of the gravitational mass for (c) temperatures in the range $[0,60] \mathrm{MeV}$ and (d) lepton fractions and entropies per baryon in the ranges $[0.2,0.4]$ and $[1,3] k_{B}$, respectively. As the temperature or the entropy per baryon (with constant lepton fraction) increases, the Kerr parameter decreases. Thermal effects conjecture that the limits introduced by the Kerr black holes [18] and neutron stars, through Eq. (3), cannot be violated. Henceforth, the gravitational collapse of a hot, uniformly rotating neutron star, constrained to mass-energy and angular momentum conservation, cannot lead to a maximally rotating Kerr black hole.

In Figures 5(e,f) we display the angular velocity as a function of the ratio $T / W$ for (e) temperatures in the range $[0,60] \mathrm{MeV}$ and (f) lepton fractions and entropies per baryon in the ranges $[0.2,0.4]$ and $[1,3] k_{B}$, respectively. In neutron stars, the point that locates the nonaxisymmetric instability, which is responsible for the emission of gravitational waves, is defined via the ratio of rotational kinetic to gravitational binding energy $T / W$. In particular, instabilities driven by gravitational radiation for models with $M_{\mathrm{gr}}=1.4 M_{\odot}$ would set in at $T / W \sim 0.08$ [21]. For sufficiently compact neutron stars or low temperatures, the nonaxisymmetric instability will set in before the mass-shedding limit is reached. The information that can be gained is that the maximum gravitational mass and the angular velocity will be lowered.

Assuming a binary neutron star merger remnant with at least a temperature of $30 \mathrm{MeV}$ or $S=1$ with $Y_{l}=0.2$, constraints are possible through the compactness parameter, the Kerr parameter, and the ratio of kinetic to gravitational 


\section{P.S. Koliogiannis, Ch.C. Moustakidis}

binding energy. After a binary neutron star merger, the remnant is a compact object consisting of neutron star matter, additionally to its high rotational rates. Considering the maximum mass configuration at the mass-shedding limit (a) the compactness parameter is constraint to $\beta_{\mathrm{rem}}^{\text {iso }} \leq 0.19$ and $\beta_{\mathrm{rem}}^{\text {ise }} \leq 0.27$, (b) the Kerr parameter is constraint to $\mathcal{K}_{\text {rem }}^{\text {iso }} \leq 0.42$ and $\mathcal{K}_{\text {rem }}^{\text {ise }} \leq 0.68$, and (c) the ratio $T / W$ is constraint to $(T / W)_{\text {rem }}^{\text {iso }} \leq 0.05$ and $(T / W)_{\text {rem }}^{\text {ise }} \leq 0.127$ (the superscripts "iso" and "ise", correspond to isothermal and isentropic profiles). Based on the above values, two conjectures can be made for the aftermath of a neutron star merger. In particular, in the isothermal case, it creates a lesser compact star than the cold neutron star with lower values of maximum gravitational mass and frequency, and stable toward dynamical instabilities, where for the isentropic aftermath, the object is comparable to the cold one [9].

\section{Concluding Remarks}

The role of thermal support in isolate neutron stars and binary neutron star merger remnants have been extensively studied with the use of thermodynamically consistent EoSs (isothermal and isentropic), and the construction of nonrotating and uniformly rotating at the mass-shedding limit axisymmetric equilibrium sequences. The astrophysical applications of the EoSs may be acceptable for a first-order study of protoneutron stars, as well as of the postmerger remnant that has survived the initial highly dynamical and strongly nonaxisymmetric postmerger evolution and settled down into a stable quasi-equilibrium state [22].

The nuclear model which applied for the construction of the EoSs, presents merits compared to other models: (a) the thermal effects (both in isothermal and isentropic profiles) have been included in a self-consistent way, (b) the model is flexible enough to produce a wide range of EoSs by properly modifying the density dependence of the symmetry energy, (c) the model reproduces microscopic calculations concerning both the SNM and the PNM, (d) the momentum dependence of the potential interaction (which is absent in the majority of the proposed models) is in accordance with the terrestrial studies and experiments of heavy-ion reactions for both low and high densities and temperatures, and (e) the model ensures the causal behavior of the EoS at high densities.

The baryon mass of a neutron star is a conservative quantity, suggesting that the temperature dependence is significant. In fact, the rising of the temperature in the core of the neutron star has as a consequence to lower the value of the baryon mass. In particular, we focus on the rapidly rotating remnant assuming its rotation at mass-shedding limit. In the cold case, the baryon mass is $3.085 M_{\odot}$, while for a hot one at $T=30 \mathrm{MeV}$ is $2.427 M_{\odot}$ and for one at $S=1$ is $3.05 M_{\odot}$. By assuming equal masses of merger components, these limits correspond to merger components of $\sim 1.5425, \sim 1.2135$, and $\sim 1.525 M_{\odot}$ baryon masses, respectively. Additionally, the immediate aftermaths of GW170817 [7] and GW190425 [8] events had created hot and rapidly rotating remnants. Al- 


\section{Thermal Support and Rapid Uniform Rotation}

though the merger remnants are born with differential rotation, the uniform rotation approach can provide us with useful insight about the EoS. In the case of GW170817 event, a remnant with a total mass of $\sim 2.7 M_{\odot}$ has been created. In correlation with the MDI+APR1 EoS, with respect to baryon mass, only cold and isentropic neutron stars with uniform rotation can support the remnant. As for the GW190425 event, the existence of the $\sim 3.7 M_{\odot}$ remnant cannot be described only by uniform rotation.

Moment of inertia informs us about the structure of the neutron star as it continuously changes its angular velocity and loses angular momentum due to radiation. Thermal support lead to the conclusion that moment of inertia, and compactness parameter, have lower values than the cold neutron star. This particular behavior is influenced by the unique interplay between the gravitational mass and the equatorial radius.

The interplay between the Kerr parameter and temperature has as a result the reduction of the Kerr parameter, setting the maximum value equal to the one of the cold neutron star. In this way, as the limit for neutron stars is lower than the one for Kerr black holes, the thermal support cannot lead a star to collapse into a maximally rotating Kerr black hole. In addition, after $\sim 1 M_{\odot}$, while in the cold configuration the Kerr parameter is approximately constant, in hot configurations is an increasing function of the gravitational mass, leading to a unique maximum value. The latter suggests the peculiar relation between the gravitational mass and angular momentum as the temperature increases.

In the gravitational collapse due to gravitational radiation, the value of the ratio $T / W$ is $\sim 0.08$ for the $M_{\mathrm{gr}}=1.4 M_{\odot}$ configuration [21]. Thermal support lead the star to lower values for the ratio $T / W$, meaning that the instabilities by gravitational radiation never occur in a postmerger remnant or a hot, rapidly rotating neutron star. In the cases where the ratio exceeds the $T / W \sim 0.08$, this value sets the limit for the maximum gravitational mass and angular velocity, lowering the latter quantities for the neutron star.

Evolutionary sequences of constant baryon mass can interpret both thermal and instability effects in neutron stars. Specifically, in the case of the Kepler frequency and the central baryon density, for temperatures $T \geq 30 \mathrm{MeV}$, the existence of a linear relation between these quantities, defines the allowed region of the pair of the central baryon density and corresponding Kepler frequency for a rotating hot neutron star at its mass-shedding limit.

Future work should include differential rotation, with emphasis on the various differential laws, and a broader set of hot microphysical EoSs. Finally, the postmerger remnant and its dynamical processes should be thoroughly investigated, as the LIGO and Virgo collaboration will provide us with more events of neutron star mergers.

For the numerical integration of the equilibrium equations we used the publicly available numerical code nrotstar from the $\mathrm{C}++$ Lorene/Nrotstar library [23] (for 


\section{P.S. Koliogiannis, Ch.C. Moustakidis}

more details see Ref. [9]; for the instability criteria see Refs. [9, 24, 25]).

\section{Acknowledgements}

Authors thank the financial support of the Bulgarian National Science Fund under contract No. KP-06-N48/1.

\section{References}

[1] M. Prakash, I. Bombaci, M. Prakash, P.J. Ellis, J.M. Lattimer, R. Knorren (1997) Phys. Rep. 2801.

[2] J.O. Goussard, P. Haensel, J.L. Zdunik (1997) Astron. Astrophys. 321822.

[3] N. Glendenning (2000) "Compact Stars: Nuclear Physics, Particle Physics, and General Relativity (Springer, Berlin).

[4] F. Weber (1996) "Pulsars as astrophysical laboratories for nuclear and particle physics" (UK: Institute of Physics, Bristol).

[5] H. Cromartie, E. Fonseca, S. Ransom, P.B. Demorest, Z. Arzoumanian et al. (2020) Nat. Astron. 472.

[6] J.W.T. Hessels, S.M. Ransom, I.H. Stairs, P.C.C. Freire, V.M. Kaspi, and F. Camile (2006) Science 3111901.

[7] B.P. Abbott, R. Abbott, T.D. Abbott et al. (2017) Phys. Rev. Lett. 119161101.

[8] B.P. Abbott, R. Abbott, T.D. Abbott et al. (2020) Astrophys. J. 892 L3.

[9] P.S. Koliogiannis, C.C. Moustakidis (2021) Astrophys. J. 91269.

[10] C.C. Moustakidis (2008) Phys. Rev. C 78054323.

[11] C.C. Moustakidis (2009) Int. J. Mod. Phys. D 181205.

[12] C.C. Moustakidis, C.P. Panos (2009) Phys. Rev. C 79045806.

[13] A. Akmal, V.R. Pandharipande, D.G. Ravenhall (1998) Phys. Rev. C 581804.

[14] R.P. Feynman, N. Metropolis, E. Teller (1949) Phys. Rev. 751561.

[15] G. Baym, C. Pethick, P. Sutherland (1971) Astrophys. J. 170299.

[16] J.M. Lattimer, F.D. Swesty (1991) Nucl. Phys. A 535331.

[17] M. Piarulli, I. Bombaci, D. Logoteta, A. Lovato, R.B. Wiringa (2020) Phys. Rev. C 101045801.

[18] K.S. Thorne (1974) Astrophys. J. 191507.

[19] P.S. Koliogiannis, C.C. Moustakidis (2020) Phys. Rev. C 101015805.

[20] D.S. Shao, S.P. Tang, X. Sheng, J.L. Jiang, Y.Z. Wang, Z.P. Jin, Y.Z. Fan, D.M. Wei (2020) Phys. Rev. D 101063029.

[21] S.M. Morsink, N. Stergioulas, S.R. Blattnig (1999) Astrophys. J. 510854.

[22] J.D. Kaplan, C.D. Ott, E.P. O’Connor, K. Kiuchi, L. Roberts, M. Duez (2014) Astrophys. J. 79019.

[23] LORENE: Langage objet pour la relativité numérique (1998) http://lorene.obspm.fr/.

[24] K. Takami, L. Rezzolla, S. Yoshida (2011) Mon. Not. Roy. Astron. Soc. Lett. 416 L1.

[25] L.R. Weih, E.R. Most, L. Rezzolla (2017) Mon. Not. Roy. Astron. Soc. Lett. 473 L126. 